Supporting Information for:

\title{
Synthesis of Chiral Alkenyl Cyclopropane Amino Acids for Incorporation into Stapled Peptides
}

\author{
Tsz Ying Yuen*a, Christopher J. Brown, ${ }^{b}$ Yaw Sing Tan, ${ }^{c}$ and Charles W. Johannes ${ }^{b}$ \\ a. Institute of Chemical and Engineering Sciences, Agency for Science, Technology and Research, 8 Biomedical Grove, Neuros, \\ \#07-01, Singapore 138665. E-mail: yuenty@ices.a-star.edu.sg \\ b. P53 Laboratory, Agency for Science, Technology and Research, 8A Biomedical Grove, \#06-06, Immunos, Singapore 138648 \\ c. Bioinformatics Institute, Agency for Science, Technology and Research, 30 Biopolis Street, \#07-01, Matrix, Singapore 138671
}

CONTENTS

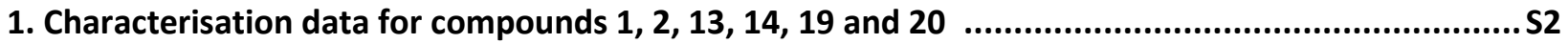

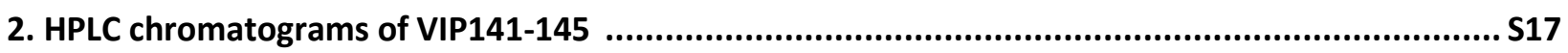


1. Characterisation data for compounds $1,2,13,14,19$ and 20

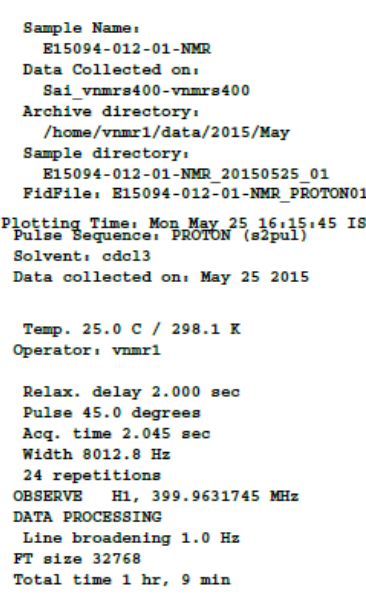

Temp. $25.0 \mathrm{C} / 298.1 \mathrm{~K}$

Operator, vamr 1

Relax, delay $2.000 \mathrm{sec}$

Pulse 45.0 degrees

W1dth $8012.8 \mathrm{~Hz}$

24 repetitions

OBSERVB H1，399.9631745 MHz

DATA PROCESSING

Line broadening $1.0 \mathrm{~Hz}$

FT size 32768

Total time $1 \mathrm{hr}, 9 \mathrm{~min}$
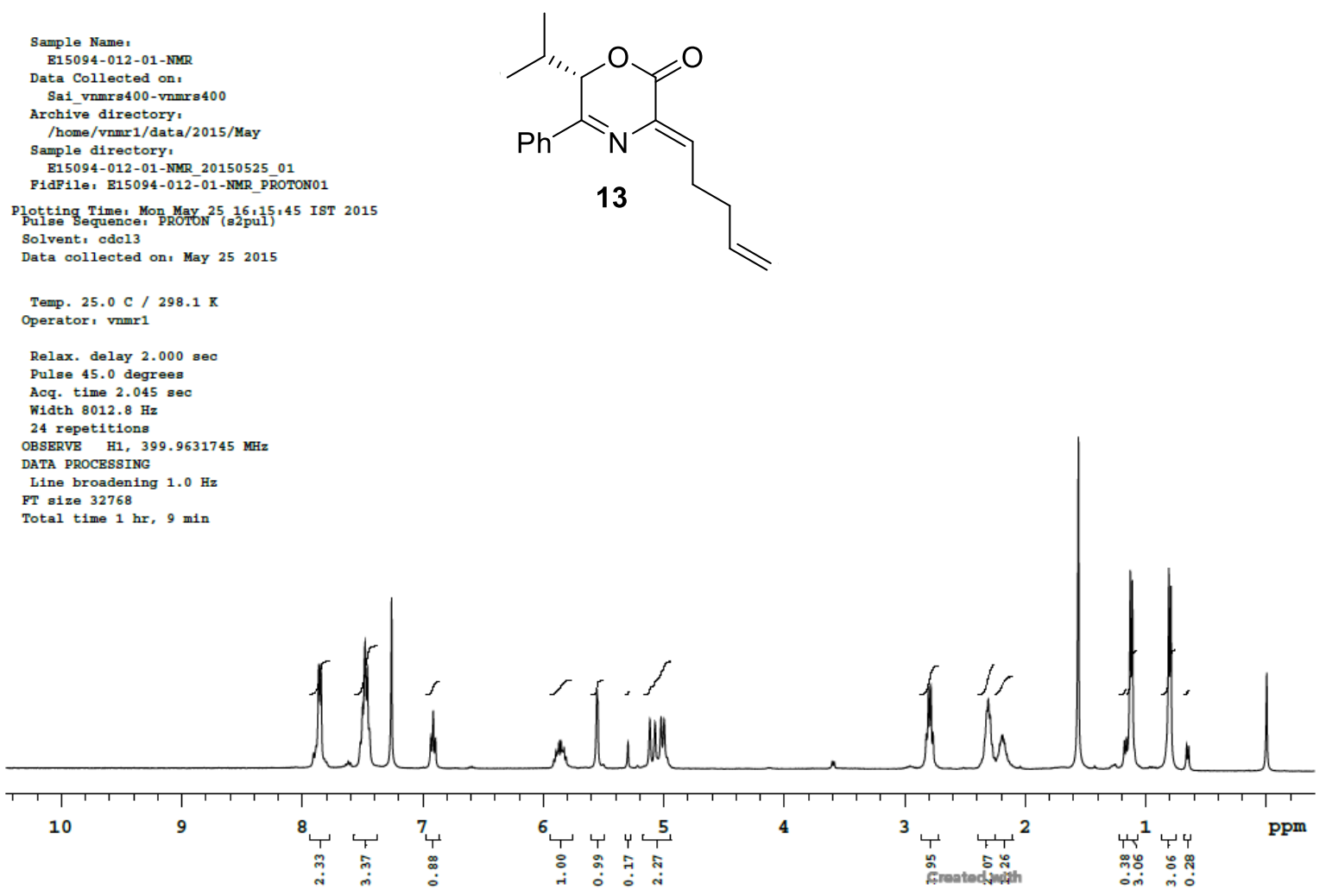

Sample Name:

B15094-012-01-NMR

Data Collected on,

Sai_vnmrs400-vnmrs40

Archive directory

/home/vnmr1/data/2015/May

Sample directory:

E15094-012-01-120150525 01

Pulse Sequence, PROTON (s2pul)

Solvent, cdc13

Solvent idell

Temp. $25.0 \mathrm{C} / 298.1 \mathrm{~K}$

Operator: vnmr1

Relax. delay 2.000 $\mathrm{sec}$

Pulse 45.0 degrees

Acq. time $2.045 \mathrm{sec}$

Width $8012.8 \mathrm{~Hz}$

28 repetitions

OBSERVB HI, 399.9631745 MHz

DATA PROCESSING

Line broadening $1.0 \mathrm{~Hz}$

Total time $1 \mathrm{hr}, 9 \mathrm{~min}$

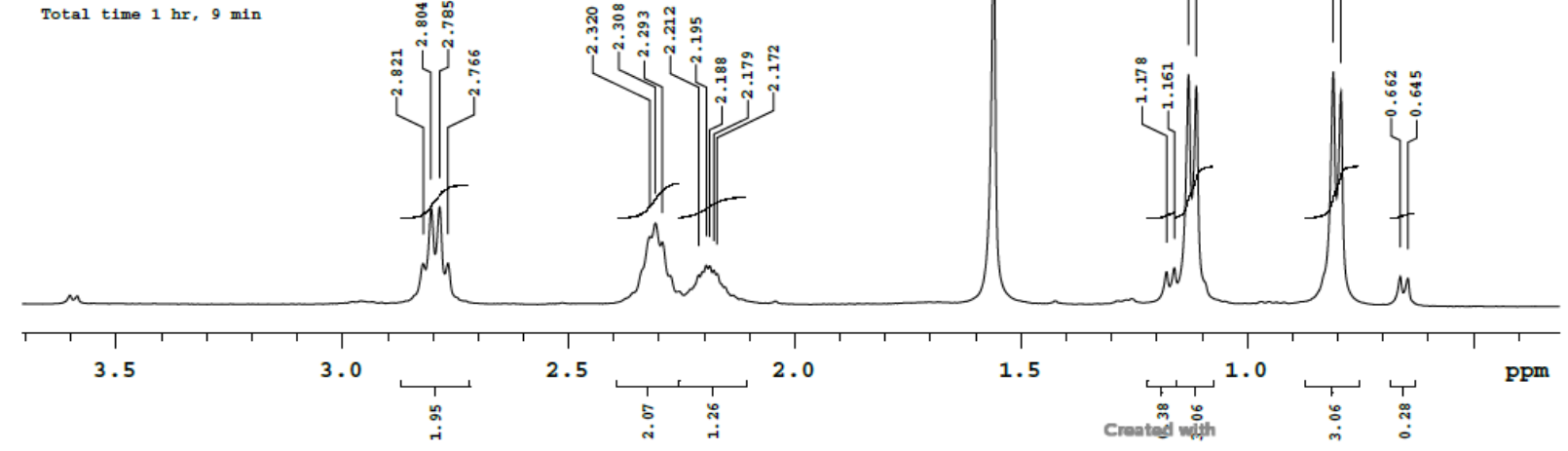




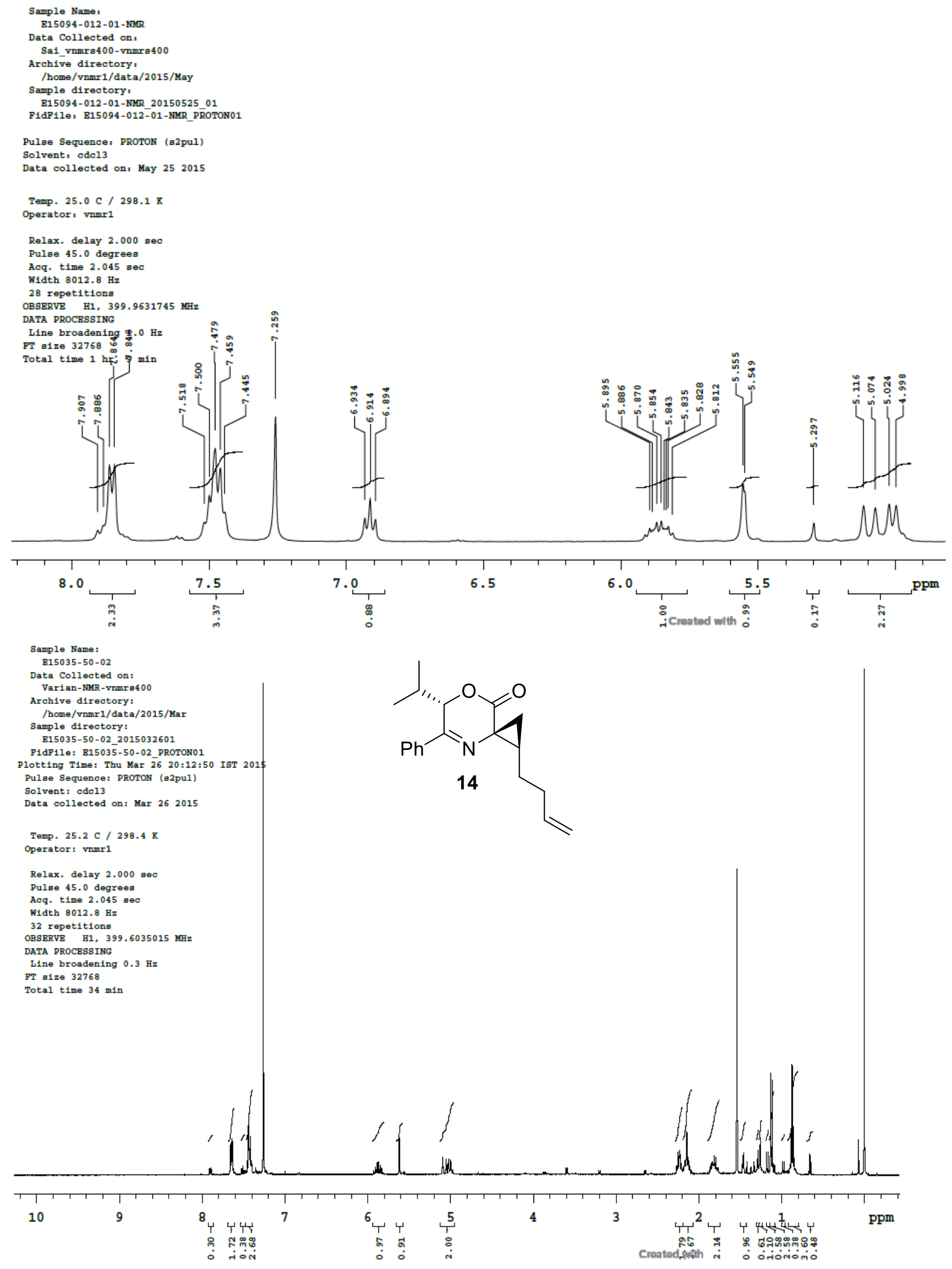




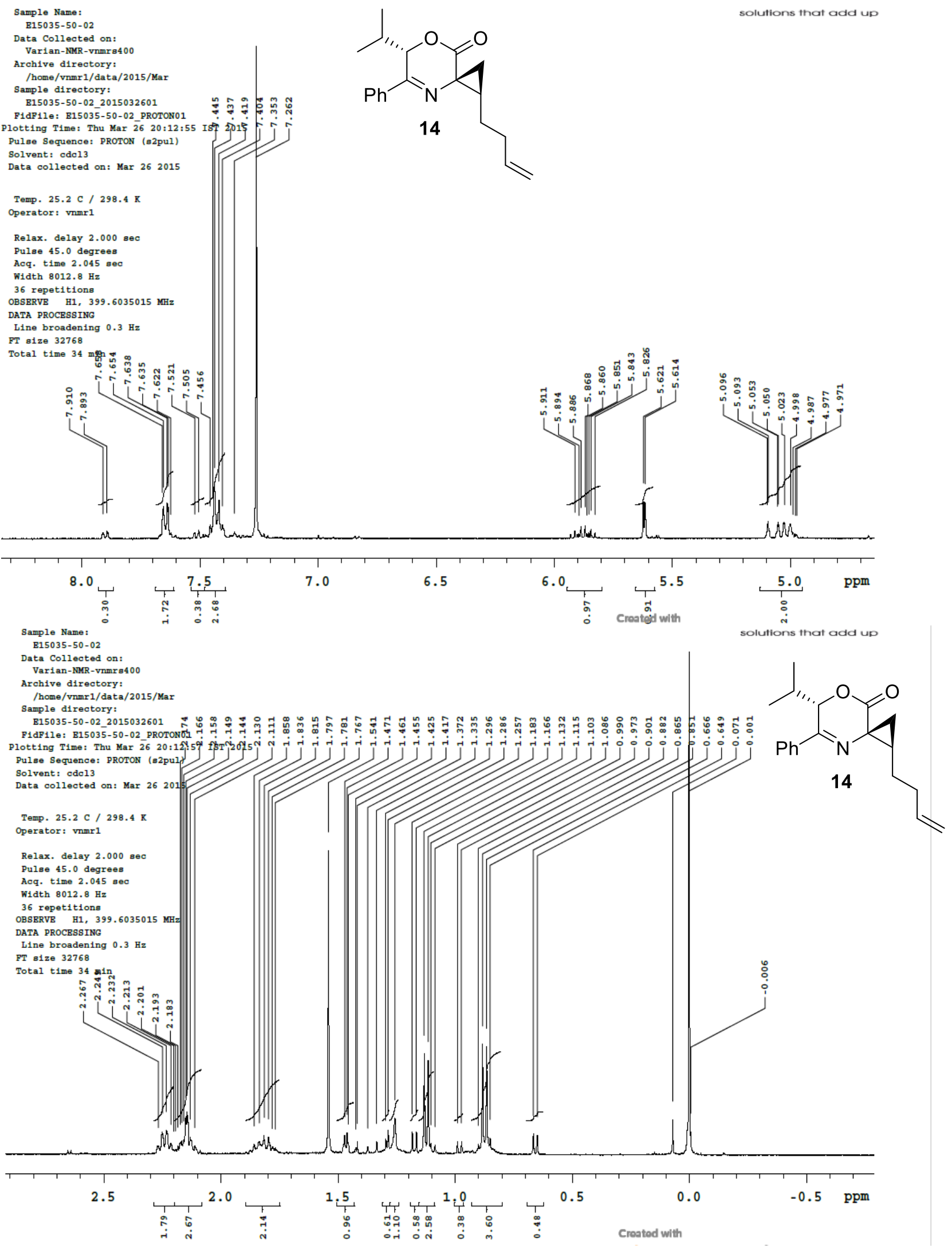


Sample Name:

E15094-63-01-NMR

Data collected on:

Sa1_vnmr 8400 -vnmrs400

Archive directory:

/home/vnmir/data/2015/Jun

ample directory:

E15094-63-01-MMR_20150617 01

lott1ng T1me: wed Jun 17 10,13:05 IST 2015

Solvent : Amso
Data collected on: Jun 172015

Temp. $25.2 \mathrm{C} / 298.4 \mathrm{~K}$

operator: vnmr

Relax. Aelay $2.000 \mathrm{sec}$

Pulse 45.0 degrees

A.

W1ath $8012.8 \mathrm{~Hz}$

OBSERVE H1, 399.9650724 MHZ

OBSERV HI,

L1ne broacening $0.9 \mathrm{Bz}$

FT s1ze 32768

Total t1me $1 \mathrm{hr}, 9 \mathrm{~min}$

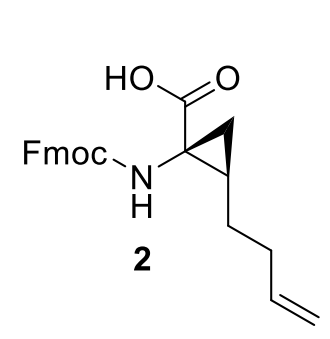

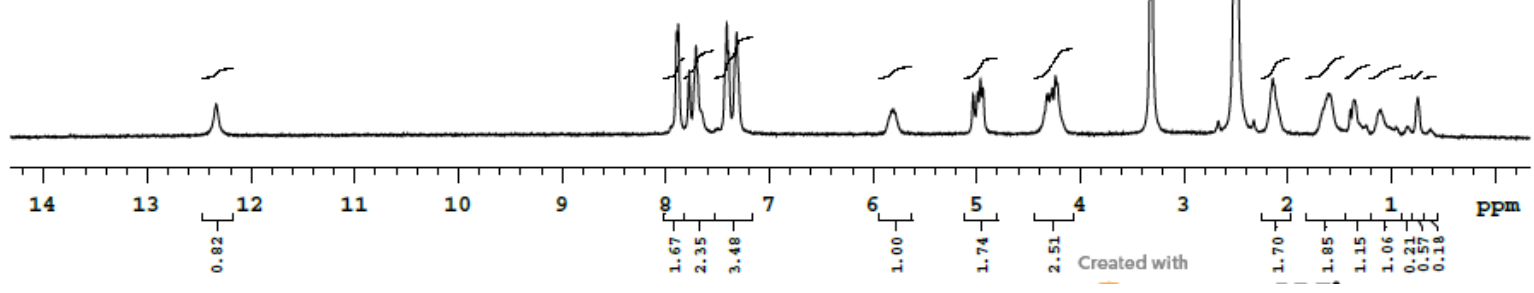

Sample Name:

B15094-63-01-NMR

Data Collected on:

Sa1_vnmr 8400 -vamrs400

Archive directory:

/home/vnmr1/đata/2015/Jun

Sample directory:

E15094-63-01-NMR 20150617

F1AP11e: E15094-63-01-NMR_PROTON01

Pulse Sequence: PROTON (s2pul)

Solvent 1 amso

Data collected on. Jun 172015

Temp. $25.2 \mathrm{C} / 298.4 \mathrm{x}$

Operator: vnmr 1

Relax. Aelay 2.000 sec

Pulse 45.0 degrees

Acq. t1me $2.045 \mathrm{se}$

w1ath $8012.8 \mathrm{~Hz}$

32 repet1tions

OBSERVE H1, 399.9650724 MB

DATA PROCESSING

Line broadening $0.9 \mathrm{~Hz}$

PT s1ze 32768

Total time $1 \mathrm{hr}, 9 \mathrm{~min}$
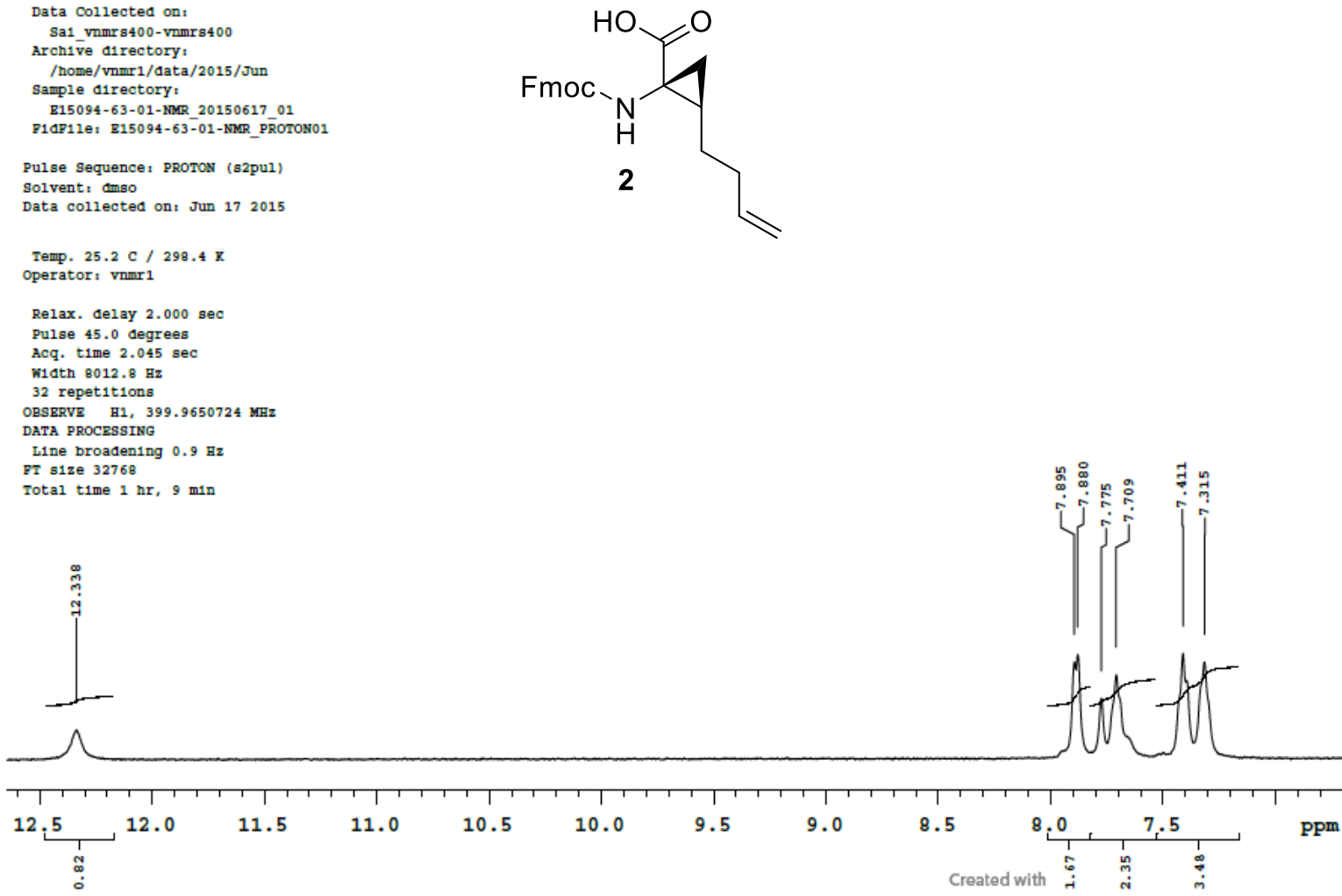


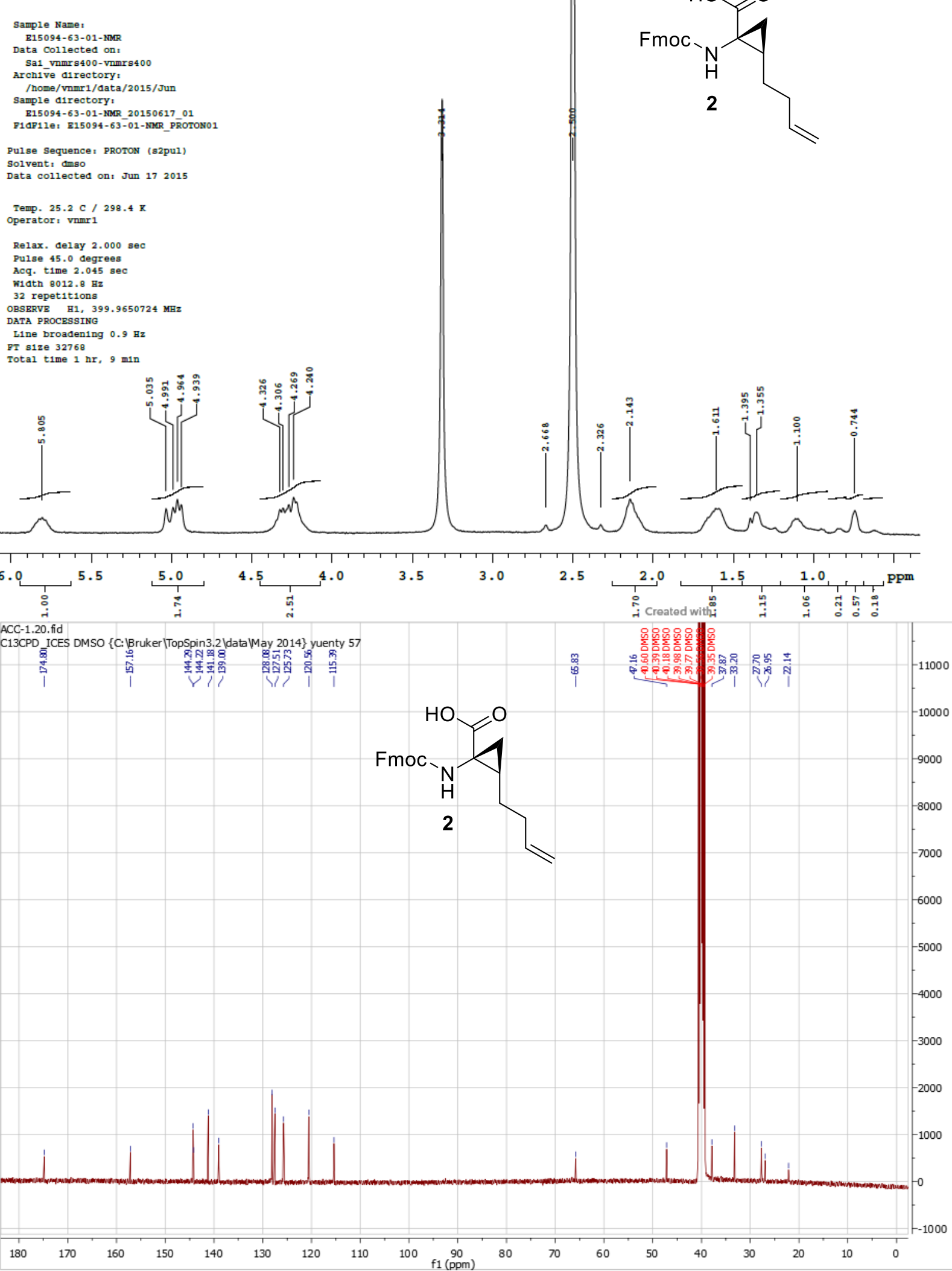




\section{HPLC REPORT}

Acquired by

Date Acquired

Sample Name

Sample ID

Data Flle

Method File

AR number
Sample Information

: Admin

: 6/16/2015 3:27:37 PM

: E15094-63-01-HPLC

: 023.lcd

: HPLC_X-Select(Formic acid)

:02-160615-019

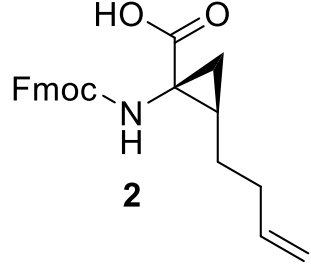

Method:- HPLC X-Select(Formic acid)

Column : X-Select CSH C18 $\left(4.6^{*} 150\right) \mathrm{mm} 3.5 \mathrm{u}$

Mobile Phase: A - Acetonitrile

Inj Volume; $5.0 \mu \mathrm{L}$,

B - $0.1 \%$ Formic acid $+5 \%$ Acetonitrile in water

Flow Rate: $1.0 . \mathrm{mL} / \mathrm{minute}$

Gradient program: Inttial hold $95 \%$ B for $1 \mathrm{mmn}$., $95 \%$ B to $0 \%$

hold till $12 \mathrm{~min}$, at $15.0 \mathrm{~min}$ B conc is $95 \%$ up to $18 \mathrm{~min}$

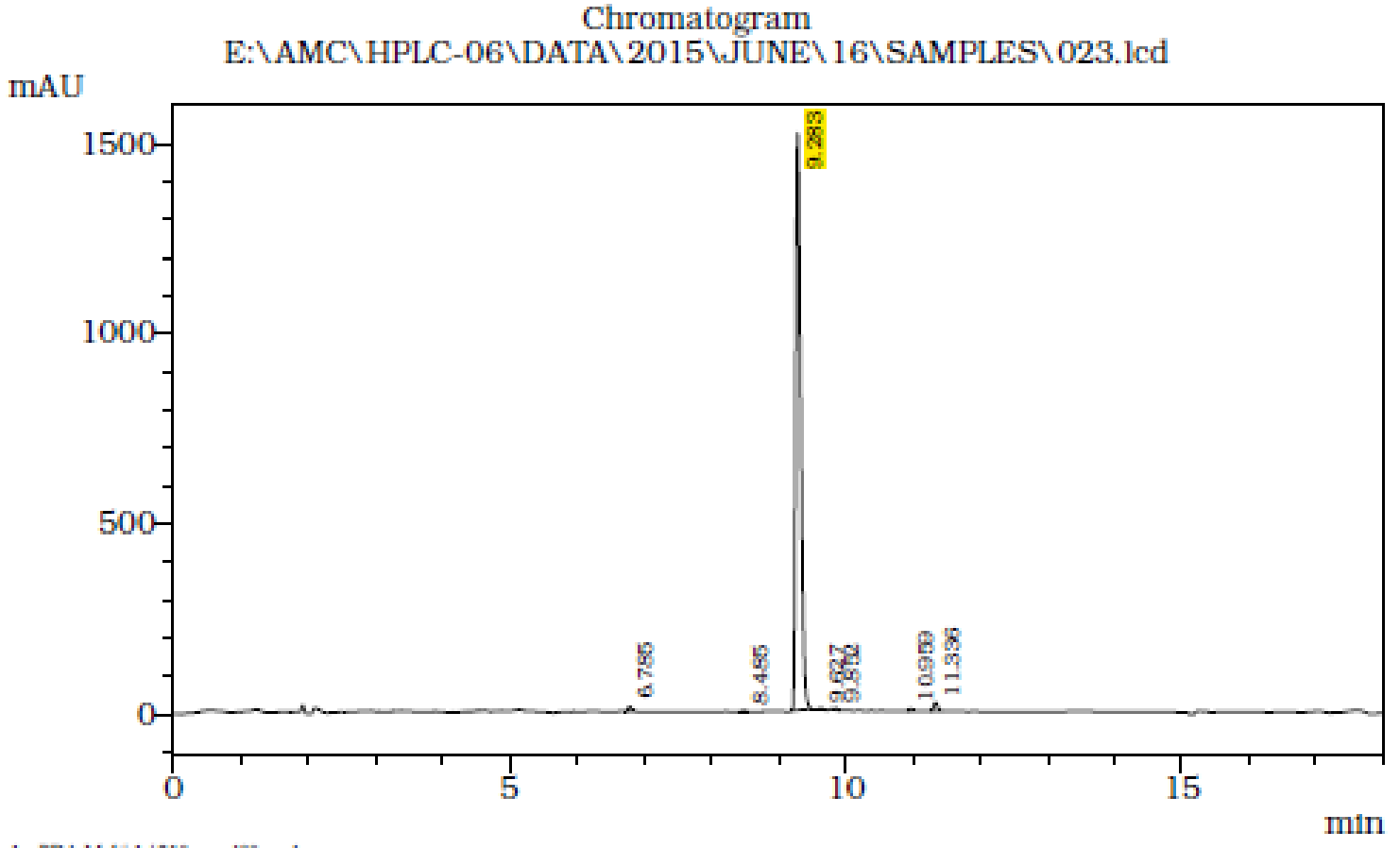

1 PDA Mula $1 / 210 \mathrm{~mm}-400 \mathrm{~mm} 4 \mathrm{~mm}$

PeakTable

PDA Chl 210nm - 400nm 4nm

\begin{tabular}{|c|c|c|c|c|}
\hline Peak\# & Ret. Time & Area & Area \% & Peak Purity Index \\
\hline 1 & 6.785 & 38725 & 0.481 & 0.99988 \\
\hline 2 & 8.485 & 12436 & 0.155 & 0.99934 \\
\hline 3 & 9.283 & 7846419 & 97.551 & 1.00000 \\
\hline 4 & 9.627 & 19967 & 0.248 & 0.99985 \\
\hline 5 & 9.852 & 16043 & 0.199 & 0.99992 \\
\hline 6 & 10.959 & 18465 & 0.230 & 0.99976 \\
\hline 7 & 11.336 & 91361 & 1.136 & 1.00000 \\
\hline Total & & 8043416 & 100.000 & \\
\hline
\end{tabular}




\section{HPLC REPORT}

\begin{tabular}{ll} 
& \multicolumn{1}{c}{ Sample Information } \\
Acquired by & $:$ Admin \\
Date Acquired & $: 7 / 1 / 201511: 03: 28 \mathrm{AM}$ \\
Sample ID & $:$ E15094-70-14 \\
Tray- & $: 2$ \\
Vial- & $: 9$ \\
Data File & $:$ CHIRAL ISOCRATIC \\
Method File &
\end{tabular}

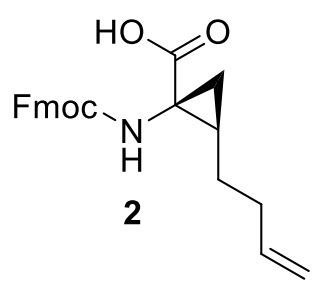

Method:-

Column: YMC CHIRALART Cellulose-SC, $250 \mathrm{~mm}^{*} 4.6 \mathrm{~mm}^{*} 5 u m$.

Mobile Phase:

A:n-Hexane+0. 1\%TFA

B:ETOH:IPA(85:15)+0. 1\%TFA

Flow rate: $1.0 \mathrm{ml} / \mathrm{min}$

Isocratic $30 \% \mathrm{~B}$.

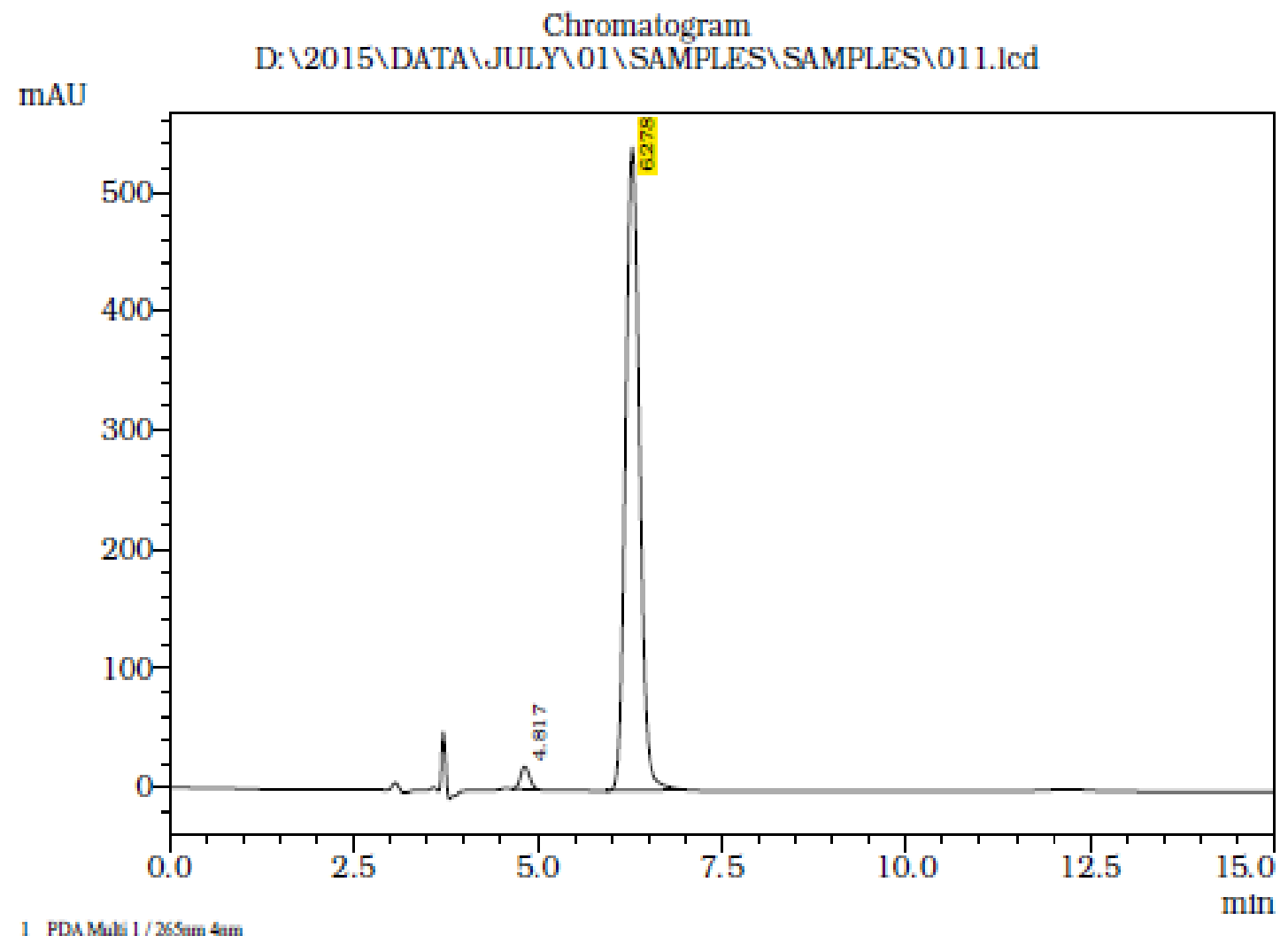

PeakTable

PDA Chl 265nm 4nm

\begin{tabular}{|r|r|r|r|r|}
\hline Peak\# & Ret. Time & \multicolumn{1}{c|}{ Area } & \multicolumn{1}{|c|}{ Area \% } & \multicolumn{1}{c|}{ Resolution } \\
\hline 1 & 4.817 & 164403 & 2.280 & 0.000 \\
\hline 2 & 6.278 & 7046769 & 97.720 & 4.898 \\
\hline Total & & 7211172 & 100.000 & \\
\hline
\end{tabular}


Compound Table

\begin{tabular}{|c|c|c|c|r|r|r|}
\hline Label & \multicolumn{1}{|c|}{ Tgt Score } & $\begin{array}{c}\text { Mass Error } \\
(\mathrm{ppm})\end{array}$ & \multicolumn{1}{|c|}{ Tgt Formula } & Obs. RT & Ref. Mass & Obs. Mass \\
\hline $\mathrm{Cpd} \mathrm{1:} \mathrm{C} 23 \mathrm{H} 23 \mathrm{~N} 04 ; 0.116$ & 95.53 & -1.71 & $\mathrm{C} 23 \mathrm{H} 23 \mathrm{~N} \mathrm{04}$ & 0.116 & 377.1627 & 377.1621 \\
\hline
\end{tabular}

\begin{tabular}{|c|c|c|c|c|c|c|}
\hline \multicolumn{1}{|c|}{ Obs. $\mathrm{m} / \mathbf{z}$} & Obs. RT & Obs. Mass & Tgt Formula & Tgt Mass & $\begin{array}{c}\text { Tgt Mass } \\
\text { Error } \\
\text { (ppm) }\end{array}$ & $\begin{array}{c}\text { Find Cpds } \\
\text { Algorithm }\end{array}$ \\
\hline 376.1549 & 0.116 & 377.1621 & C23 H23 N 04 & 377.1627 & -1.71 & Find by Formula \\
\hline
\end{tabular}

Compound Chromatograms

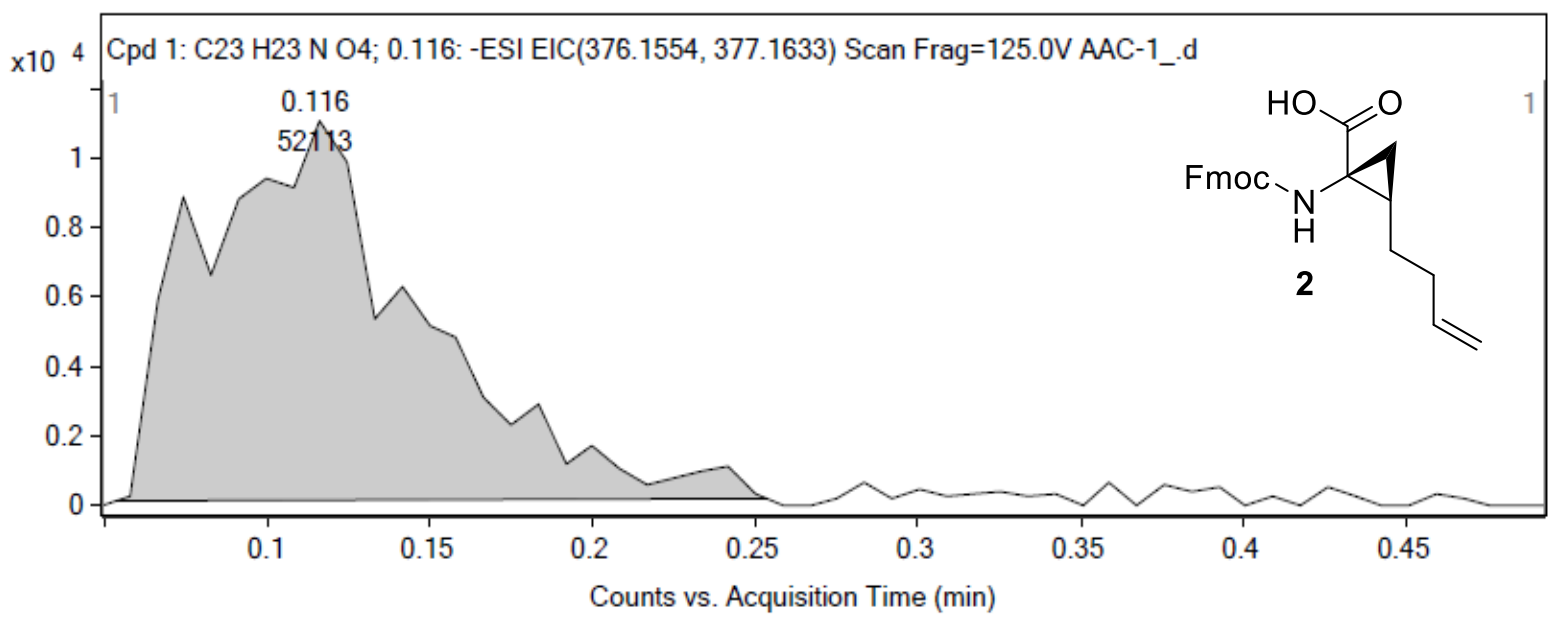

Sample Name

B15094-080-02-NMR

Data Collected on.

Sai_vnmr8400-vnmrs 400

Archive directory

/home/vnmr1/data/2015/Jun

Sample directory

E15094-080-02-NMR 20150626 01

PidPile: B15094-080-02-NMR PR̃OTON01

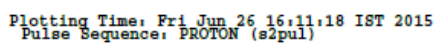

Solvent; odc13

Data collected on, Jun 262015

Temp. $25.0 \mathrm{C} / 298.1 \mathrm{~K}$

Operator : vamr 1

Relax. delay $2.000 \mathrm{sec}$

Pulse 45.0 degrees

Acq. time $2.045 \mathrm{gec}$

Width $8012.8 \mathrm{~Hz}$

16 retition

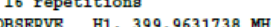

OBSERVB H1,

Line broadening $0.9 \mathrm{~Hz}$

Line broaden
FT gize 32768

Total time $34 \mathrm{~min}$
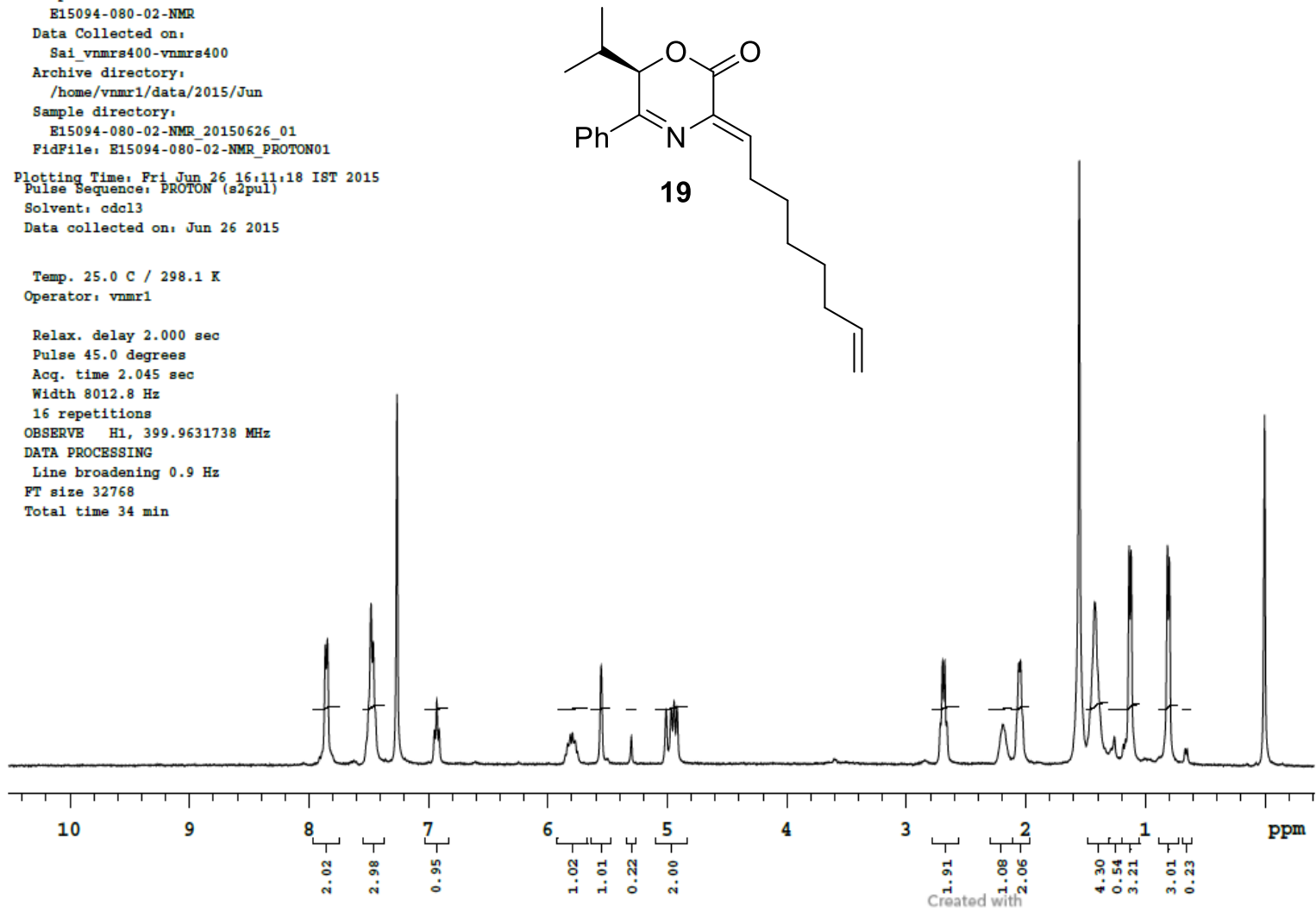


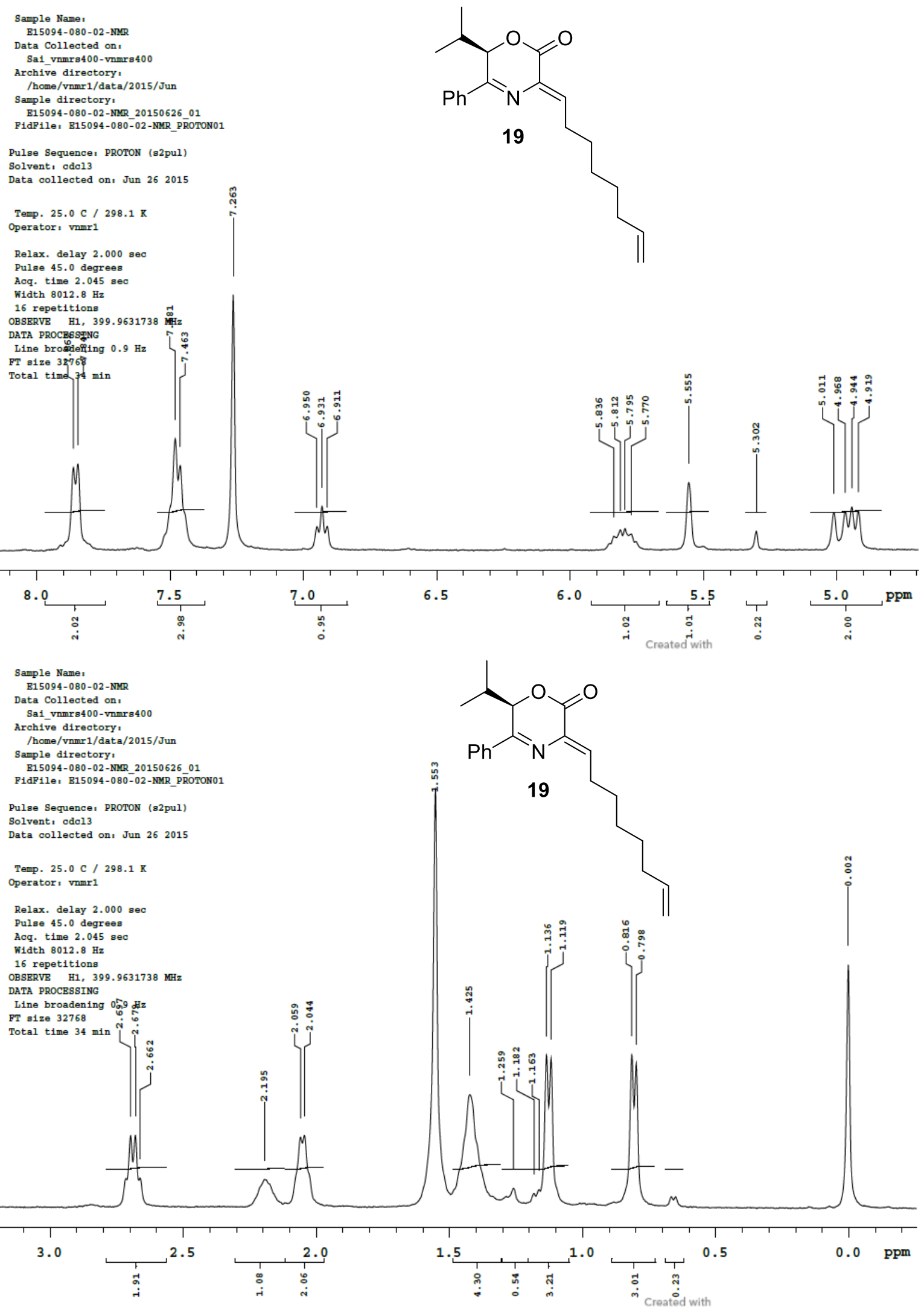




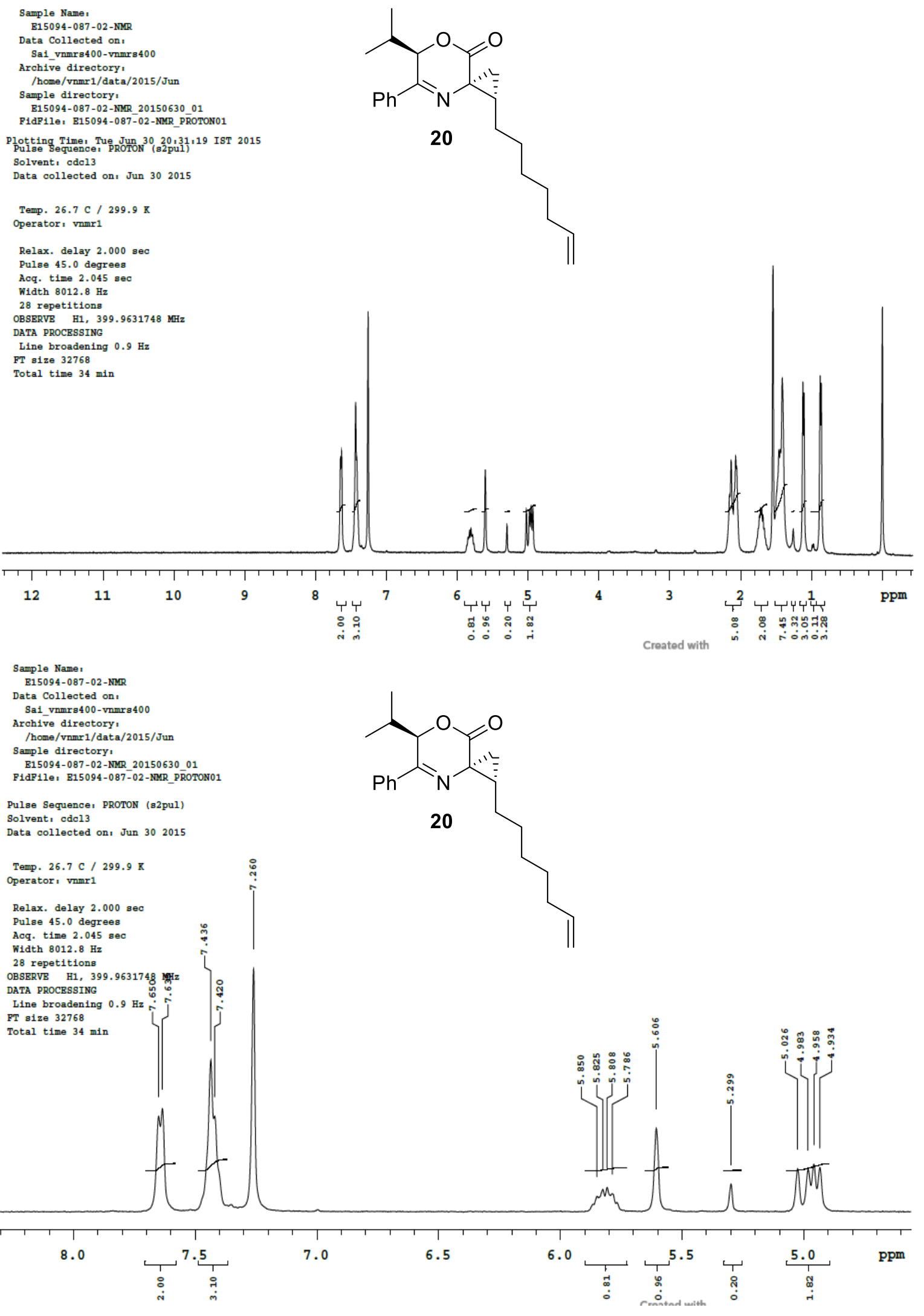



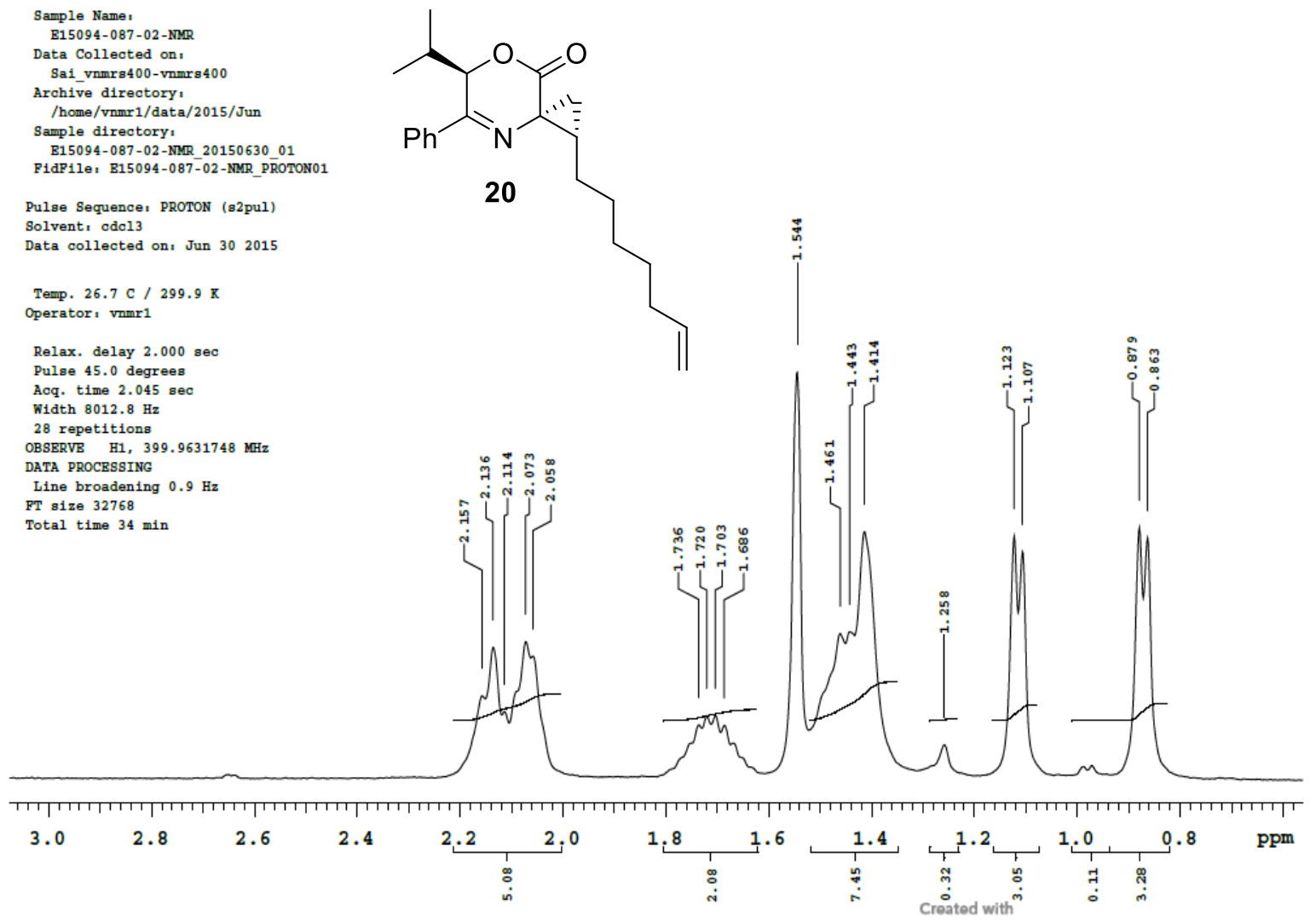

Sample Name:

B15094-82-03-NMR

Data Collected on:

Sai_vnmrs400-vnmrs

archive directory

/home/vnmr1/data/2015/Jun

E15094-82-03-Min

Fidpile. $15094-82-03-150630$ - 01

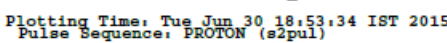

Solvent, dmso

Data collected on, Jun 302015

Temp. $28.0 \mathrm{C} / 301.1 \mathrm{~K}$

Operator: vamir

Relax. delay $2.000 \mathrm{sec}$

Pulse 45.0 degrees

Acq. time 2.045

Width $8012.8 \mathrm{~Hz}$

OBSERVB H1, 399.9650732 MHz

DSER B HE,

Line broadening $0.9 \mathrm{~Hz}$

FT size 32768

Total time $34 \mathrm{~min}$

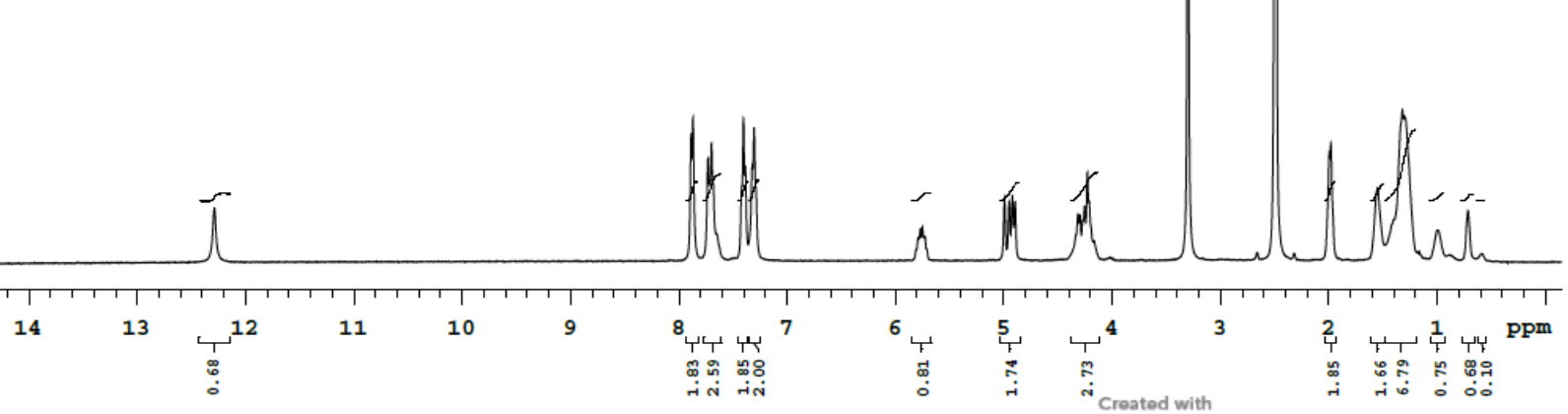




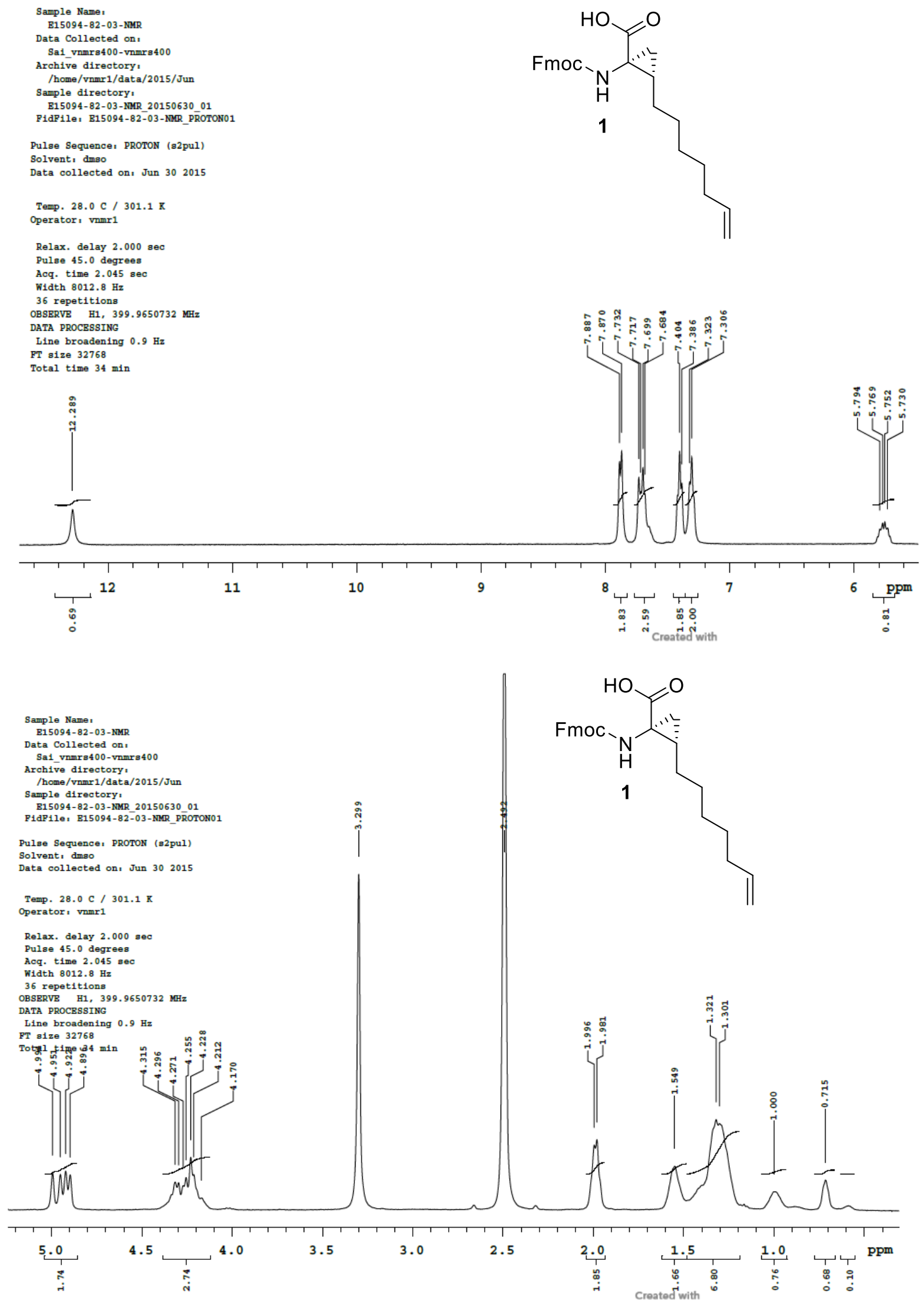




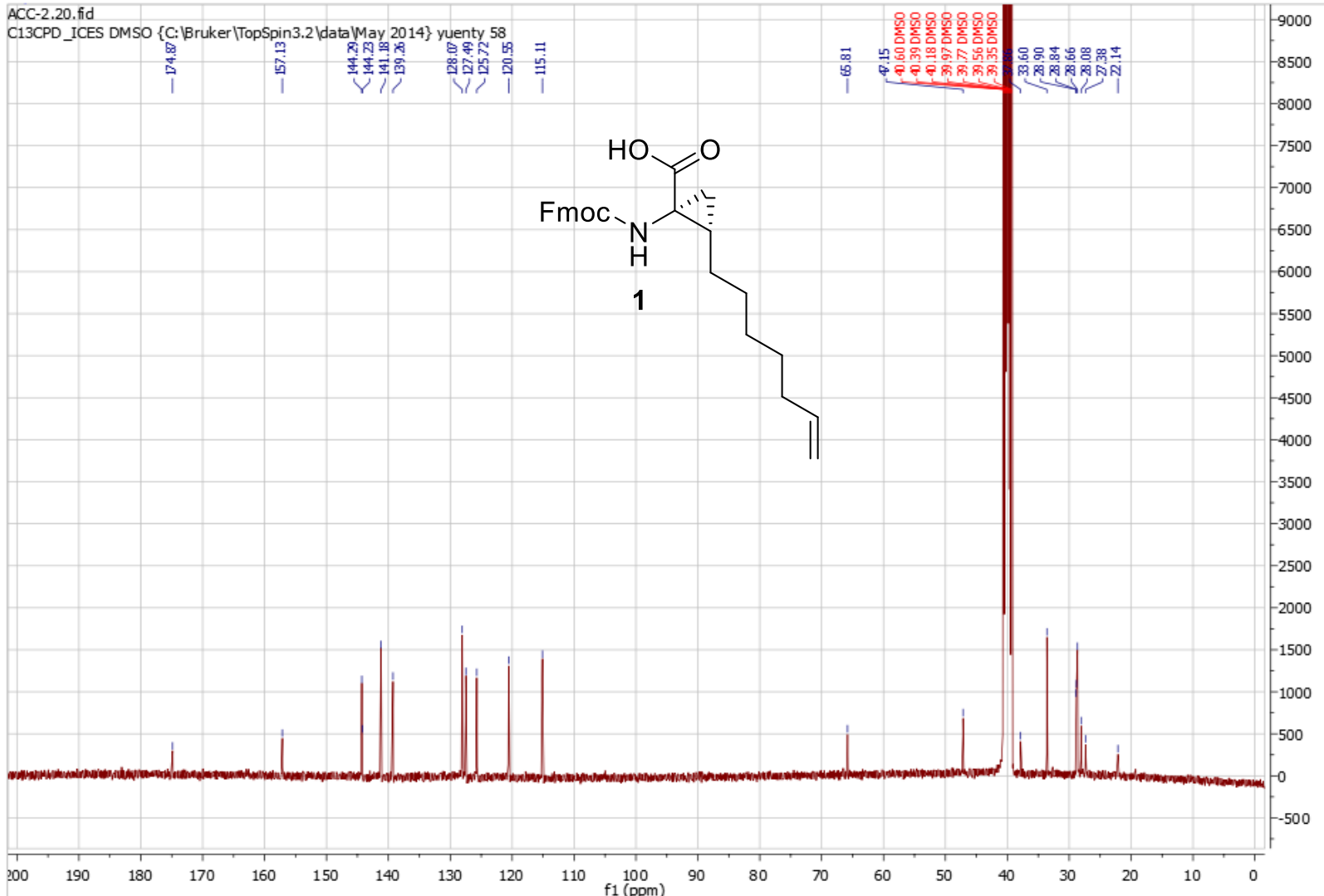




\title{
HPLC REPORT
}

\author{
Acquired by \\ Date Acquired \\ : Admin \\ Sample Name \\ : 6/30/2015 3:52:00 PM \\ Sample ID \\ Data File \\ : E15094-82-03 \\ : 027.lod \\ : HPLC_X-Select(Formic acid) \\ :02-300615-10
}

Sample Information

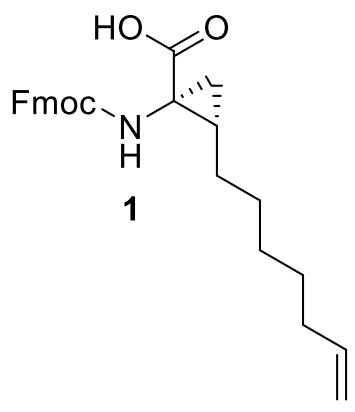

Method:- HPLC X-Select(Formic acid)

Column : X-Select CSH C18 $\left(4.6^{*} 150\right) \mathrm{mm} 3.5 \mathrm{u}$

Mobile Phase: A - Acetonitrile

$$
\text { B - } 5 \% \text { Phase A + 0.1\% Formic ac1d + water }
$$

Inj Volume; 5.0pL,

Flow Rate: $1.0 . \mathrm{mL} / \mathrm{m}$ mute

Gradient program: Intial hold $95 \%$ B for 1 min., $95 \%$ B to $0 \%$ B in 8 minute, hold till $12 \mathrm{~min}$, at $15.0 \mathrm{~min}$ B conc is $95 \%$ up to $18 \mathrm{~min}$

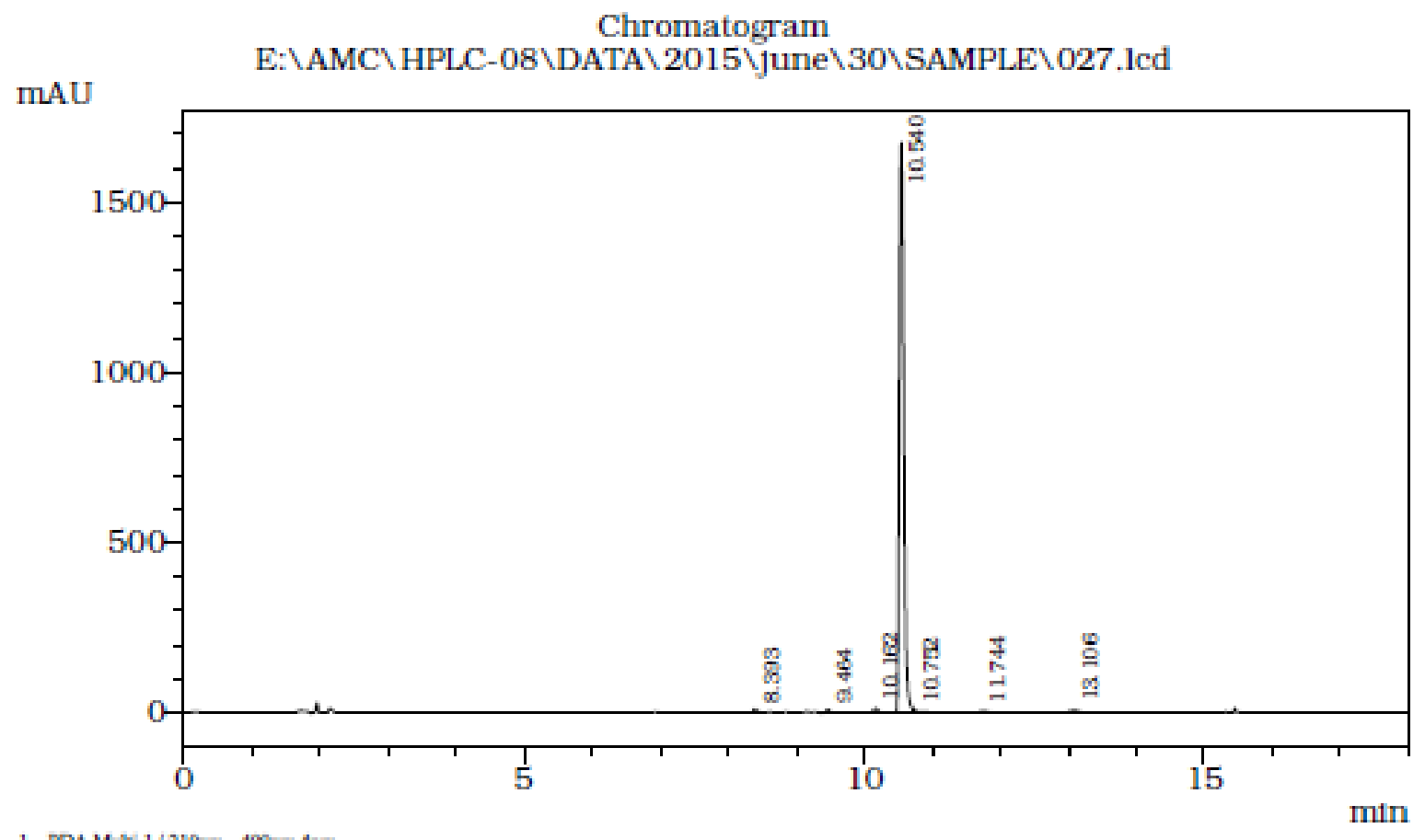

1 PDA Mulis $1 / 210 \mathrm{~km}-400 \mathrm{~mm} 4 \mathrm{em}$

PeakTable

PDA Ch1 210nm - 400nm 4nm

\begin{tabular}{|c|c|c|c|c|}
\hline Peak\# & Ret Time & Area & Area \% & Peak Purity Index \\
\hline 1 & 8.393 & 26280 & 0.313 & 0.99966 \\
\hline 2 & 9.464 & 34882 & 0.416 & 0.21524 \\
\hline 3 & 10.162 & 39407 & 0.470 & 0.64665 \\
\hline 4 & 10.540 & 8209094 & 97.889 & 0.99411 \\
\hline 5 & 10.752 & 15758 & 0.188 & 0.99920 \\
\hline 6 & 11.744 & 22326 & 0.266 & 0.99966 \\
\hline 7 & 13.106 & 38414 & 0.458 & 0.99981 \\
\hline Total & & 8386161 & 100.000 & \\
\hline
\end{tabular}




\section{HPLC REPORT}

\begin{tabular}{ll} 
& \multicolumn{1}{c}{ Sample Information } \\
Acquired by & $:$ Admin \\
Date Acquired & $: 7 / 1 / 201510: 16: 49$ AM \\
Sample ID & $:$ E15094-82-03 \\
Tray- & $: 8$ \\
Vial- & $:$ O08.Icd \\
Data File & $:$ CHIRAL ISOCRATIC \\
Method File & \\
Method:- &
\end{tabular}

Column: YMC CHIRALART Cellulose-SC, $250 \mathrm{~mm}^{*} 4.6 \mathrm{~mm}^{*} 5 \mathrm{um}$.<smiles>C=CCCCCCCC(C)C(=O)O</smiles>

Mobile Phase:

A:n-Hexane+0.1\%TFA

B:ETOH:IPA(85:15)+0. 1\%TFA

Flow rate: $1.0 \mathrm{ml} / \mathrm{min}$

Isocratic $30 \% \mathrm{~B}$.

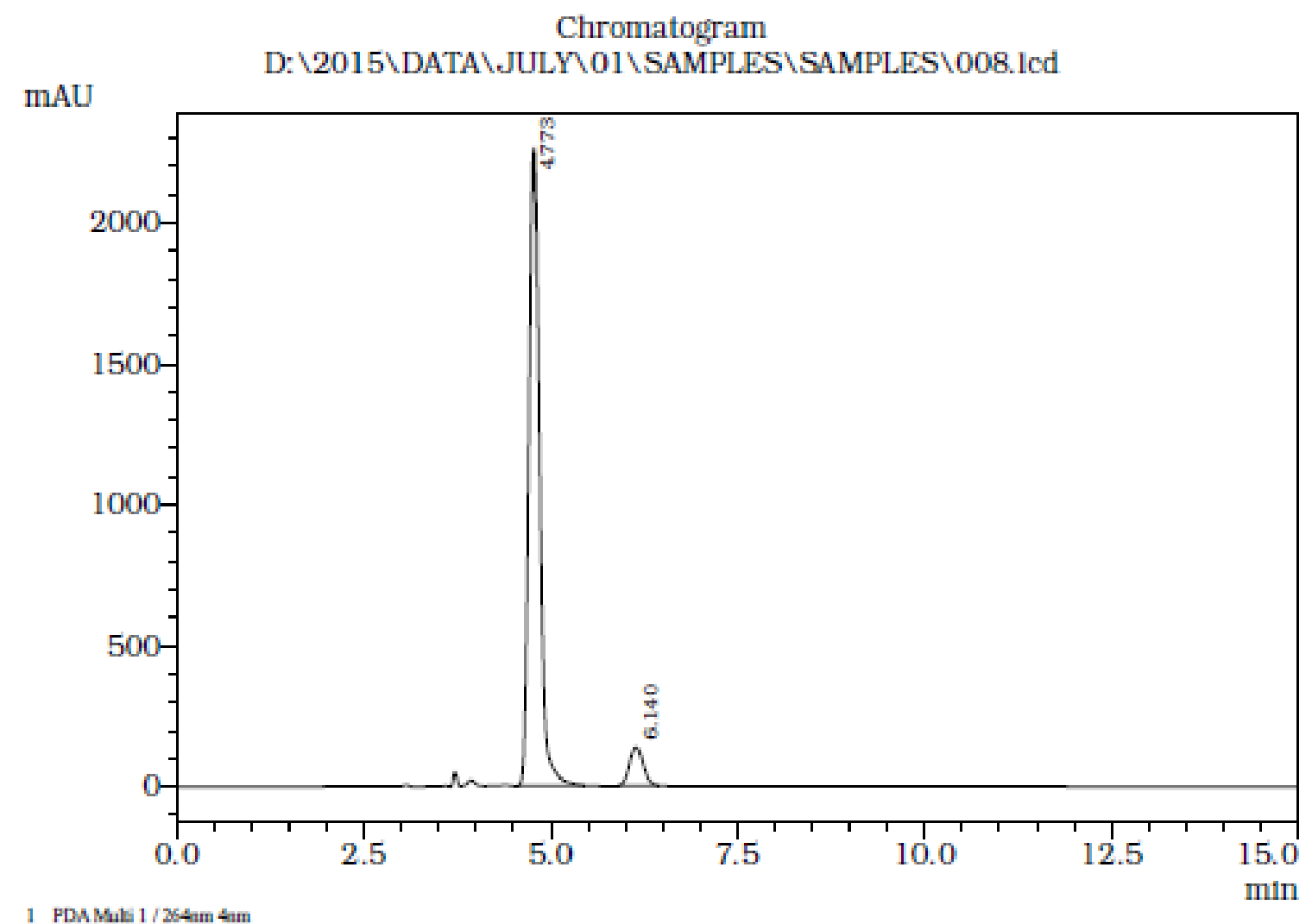

PeakTable

PDA Chl 264nm 4nm

\begin{tabular}{|r|r|r|r|r|}
\hline Peak\# & Ret. Time & \multicolumn{1}{c|}{ Area } & \multicolumn{1}{c|}{ Area \% } & Resolution \\
\hline 1 & 4.773 & 21640022 & 92.636 & 0.000 \\
\hline 2 & 6.140 & 1720213 & 7.364 & 4.543 \\
\hline Total & & 23360236 & 100.000 & \\
\hline
\end{tabular}




Compound Table
\begin{tabular}{|c|c|c|c|c|c|c|}
\hline Label & Tgt Score & $\begin{array}{c}\text { Mass Error } \\
(\mathrm{ppm})\end{array}$ & Tgt Formula & Obs. RT & Ref. Mass & Obs. Mass \\
\hline Cpd 1: C $26 \mathrm{H} 29 \mathrm{~N} 04 ; 0.097$ & 67.25 & 3.2 & $\mathrm{C} 26 \mathrm{H} 29 \mathrm{~N} \mathrm{04}$ & 0.097 & 419.2097 & 419.211 \\
\hline
\end{tabular}

\begin{tabular}{|c|c|c|c|c|c|c|}
\hline Obs. $\mathrm{m} / \mathbf{z}$ & Obs. RT & Obs. Mass & Tgt Formula & Tgt Mass & $\begin{array}{c}\text { Tgt Mass } \\
\text { Error } \\
\text { (ppm) }\end{array}$ & $\begin{array}{c}\text { Find Cpds } \\
\text { Algorithm }\end{array}$ \\
\hline 418.2032 & 0.097 & 419.211 & C26 H29 N 04 & 419.2097 & 3.2 & Find by Formula \\
\hline
\end{tabular}

Compound Chromatograms

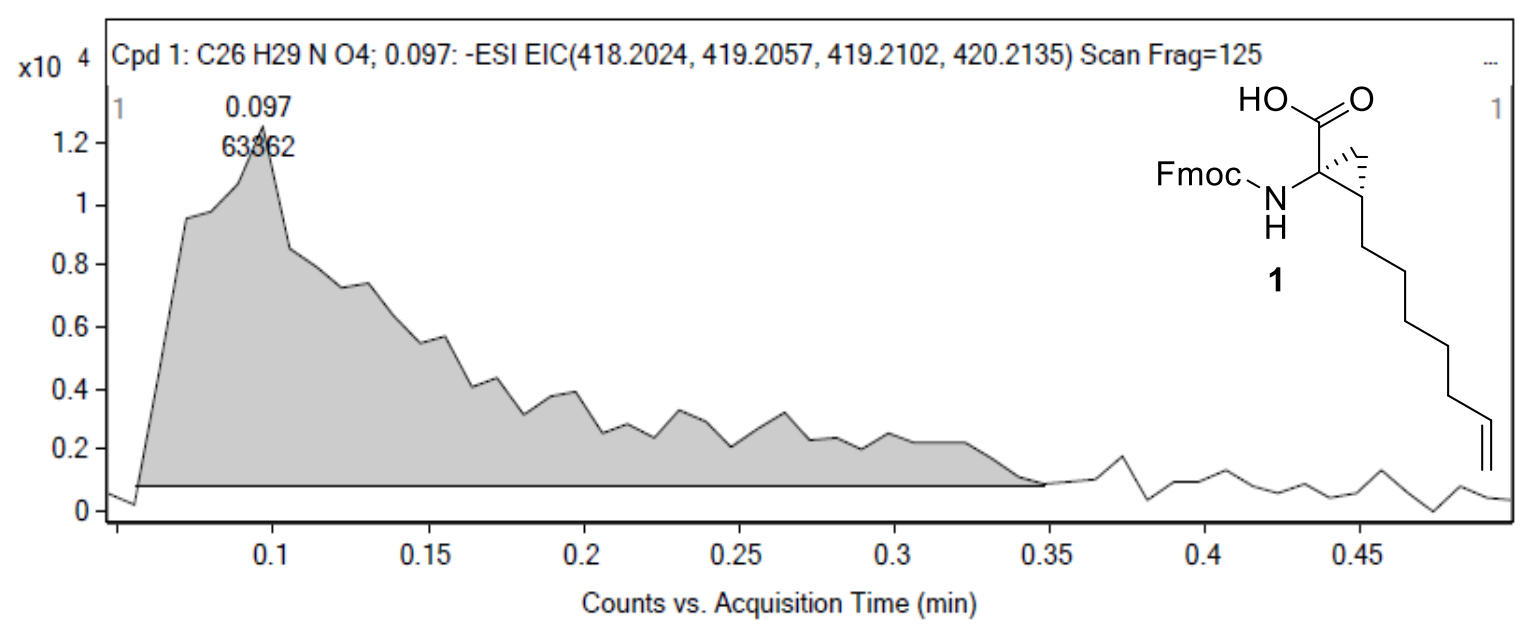

\section{HPLC chromatograms of VIP141-145}

\begin{tabular}{|c|c|c|c|c|c|}
\hline Peptide & Calculated mass & Observed mass* & RP-HPLC gradient & Purity (\%) & $\begin{array}{l}\text { Isolated peptide } \\
\text { isomeric ratio }\end{array}$ \\
\hline VIP141 & 1459.78 & $1460[\mathrm{M}-\mathrm{H}]$ & $30-70 \%$ ACN & 97.2 & undefined \\
\hline VIP142 & 1459.78 & $1459[\mathrm{M}-\mathrm{H}]$ & $30-70 \%$ ACN & 84.0 & $1: 1$ \\
\hline VIP143 & 1473.79 & $1474[\mathrm{M}-\mathrm{H}]$ & $30-70 \%$ ACN & 96.6 & $1: 1.4$ \\
\hline VIP144 & 2092.11 & $\begin{array}{c}1045[\mathrm{M} / 2-\mathrm{H}] \\
1103[\mathrm{M}+\mathrm{TFA} / 2-\mathrm{H}]\end{array}$ & $30-70 \%$ ACN & 93.1 & undefined \\
\hline VIP145 & 2106.13 & $\begin{array}{c}1053[\mathrm{M} / 2-\mathrm{H}] \\
1109[\mathrm{M}+\mathrm{TFA} / 2-\mathrm{H}]\end{array}$ & $30-70 \%$ ACN & 94.2 & $1.1: 1$ \\
\hline
\end{tabular}

*The reported MS data is for the $E / Z$ peptide mixture. 

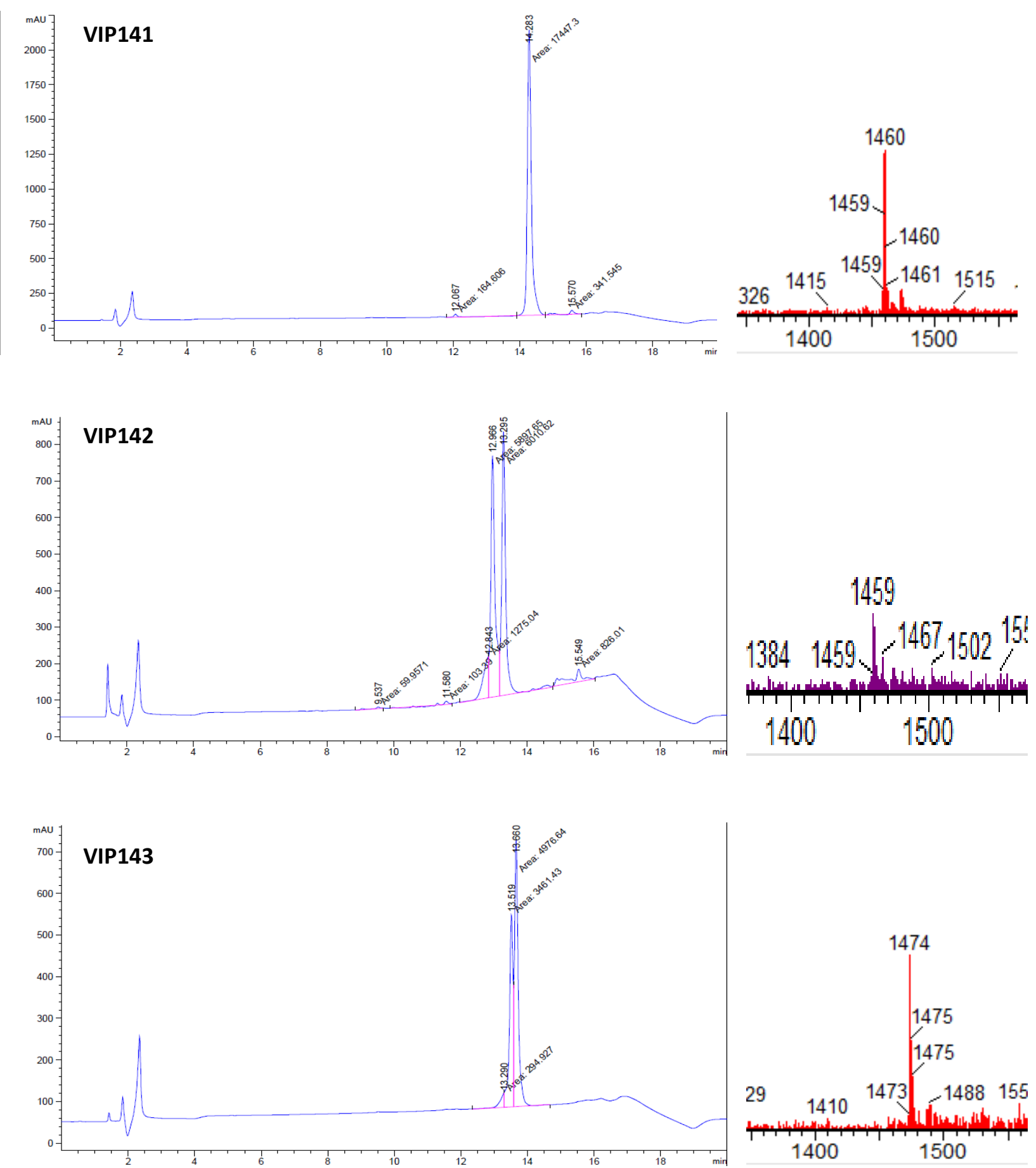

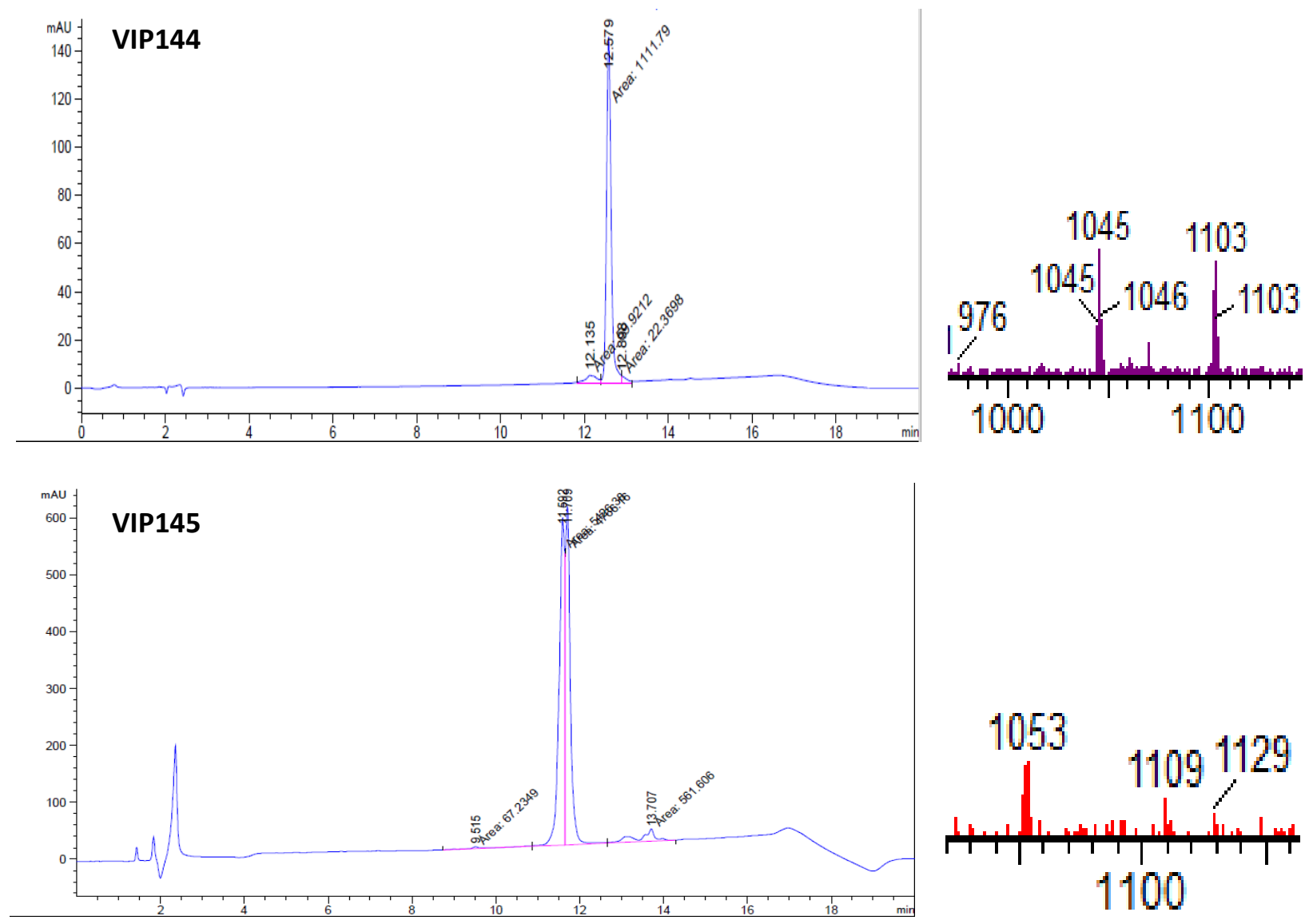